\title{
Random $k$-SAT: the limiting probability for satisfiability for moderately growing $k$
}

\author{
Amin Coja-Oghlan* \\ Alan Frieze $^{\dagger}$ \\ Department of Mathematical Sciences, \\ Carnegie Mellon University, \\ Pittsburgh PA 15213, USA. \\ acoghlaneinf.ed.ac.uk, alan@random.math.cmu.edu \\ Submitted: Sep 11, 2007; Accepted: Jan 17, 2008; Published: Feb 4, 2008 \\ Mathematics Subject Classification: 05C88
}

\begin{abstract}
We consider a random instance $I_{m}=I_{m, n, k}$ of $k$-SAT with $n$ variables and $m$ clauses, where $k=k(n)$ satisfies $k-\log _{2} n \rightarrow \infty$. Let $m=2^{k}(n \ln 2+c)$ for an absolute constant c. We prove that

$$
\lim _{n \rightarrow \infty} \operatorname{Pr}\left(I_{m} \text { is satisfiable }\right)=e^{-e^{-c}} .
$$
\end{abstract}

\section{Introduction}

An instance of $k$-SAT is defined by a set of variables, $V=\left\{x_{1}, x_{2}, \ldots, x_{n}\right\}$ and a set of clauses $C_{1}, C_{2}, \ldots, C_{m}$. We will let clause $C_{i}$ be a sequence $\left(\lambda_{i, 1}, \lambda_{i, 2}, \ldots, \lambda_{i, k}\right)$ where each literal $\lambda_{i, l}$ is a member of $L=V \cup \bar{V}$ where $\bar{V}=\left\{\bar{x}_{1}, \bar{x}_{2}, \ldots, \bar{x}_{n}\right\}$. In our random model, each $\lambda_{i, l}$ is chosen independently and uniformly from $L .{ }^{1}$ We denote the resulting random instance by $I_{m}=I_{m, n, k}$.

\footnotetext{
${ }^{*}$ Supported by DFG COJ 646.

${ }^{\dagger}$ Supported in part by NSF grant CCF-0502793

${ }^{1}$ We are aware that this allows clauses to have repeated literals or instances of $x, \bar{x}$. The focus of the paper is on $k=O(\ln n)$, although the main result is valid for larger $k$. Thus most clauses will not have repeated clauses or contain a pair $x, \bar{x}$.
} 
Random $k$-SAT has been well studied, to say the least, see the references in [6]. If $k=2$ then it is known that there is a satisfiability threshold at around $m=n$. More precisely, if $\epsilon>0$ is fixed and $I$ is a random instance of 2-SAT then

$$
\lim _{n \rightarrow \infty} \operatorname{Pr}\left(I_{m, n, 2} \text { is satisfiable }\right)= \begin{cases}1 & m \leq(1-\epsilon) n \\ 0 & m \geq(1+\epsilon) n\end{cases}
$$

Thus random 2-SAT is now pretty much understood.

For $k \geq 3$ the story is very different. It is now known that a threshold for satisfiability exists in some (not completely satisfactory) sense, Friedgut [5]. There has been considerable work on trying to find estimates for this threshold in the case $k=3$, see the references in [6]. Currently the best lower bound for the threshold is 3.52, due to Hajiaghayi and Sorkin [7] and Kaporis, Kirousis, and Lalas [8]. Upper bounds have been pursued with the same vigour. Currently the best upper bound for the threshold is 4.506 due to Dubois, Boufkhad and Mandler [4].

Building upon Achlioptas and Moore [1], Achlioptas and Peres [3] made a considerable breakthrough for $k \geq 4$. Using a sophisticated secnd moment argument, they showed that if $m \leq$ $\left(2^{k} \ln 2-t_{k}\right) n$ then whp a random instance of $k$-SAT $I_{m, n, k}$ is satifiable, where $t_{k}=O(k)$. Since a simple first moment argument shows that $I_{m, n, k}$ is unsatisfiable if $m>\left(2^{k} \ln 2+o(1)\right) n$, they have obtained an asymptotically tight estimate of the threshold for satisfiability when $k$ is a large constant.

An earlier paper by Frieze and Wormald [6] showed the following: Suppose $\omega=k-\log _{2} n \rightarrow$ $\infty$. Let

$$
m_{0}=-\frac{n \ln 2}{\ln \left(1-2^{-k}\right)}=2^{k}\left(n \ln 2+O\left(2^{-k}\right)\right) .
$$

so that $2^{n}\left(1-\frac{1}{2^{k}}\right)^{m_{0}}=1$ and let $\epsilon=\epsilon(n)>0$ be such that $\epsilon n \rightarrow \infty$. Let $I_{m}$ be a random instance of $k$-SAT with $n$ variables and $m$ clauses. Then

$$
\lim _{n \rightarrow \infty} \operatorname{Pr}\left(I_{m} \text { is satisfiable }\right)= \begin{cases}1 & m \leq(1-\epsilon) m_{0} \\ 0 & m \geq(1+\epsilon) m_{0} .\end{cases}
$$

The aim of this short note is to tighten (2) and prove the following.

Theorem 1. Suppose $\omega=k-\log _{2} n \rightarrow \infty$ but $\omega=o(\ln n)$. Let $m=2^{k}(n \ln 2+c)$ for an absolute constant $c$. Then

$$
\lim _{n \rightarrow \infty} \operatorname{Pr}\left(I_{m} \text { is satisfiable }\right)=1-e^{-e^{-c}}
$$

Theorems such as this are common in random graphs and usually indicate that the threshold for a certain property $\mathcal{P}_{1}$ depends on the occurrence of some much simpler property $\mathcal{P}_{2}$, a classic example being the case where $\mathcal{P}_{1}$ is Hamiltonicity and $\mathcal{P}_{2}$ is minimum degree at least two. Here there does not seem to be a good candidate for $\mathcal{P}_{2}$. 


\section{Proof of Theorem 1}

Let $X_{m}=X\left(I_{m}\right)$ denote the number of satisfying assignments for instance $I_{m}$. Suppose that $k=\log _{2} n+\omega$. Let $m_{0} \sim 2^{k} n \ln 2$ be as in (1) and $m_{1}=m_{0}-2^{k} \gamma$, where $\gamma=\ln \omega$. The following results can be deduced from the calculations in [6]: If $\sigma_{1}, \sigma_{2}$ are two assignments to the variables $V$, then $h\left(\sigma_{1}, \sigma_{2}\right)$ is the number of indices $i$ for which $\sigma_{1}(i) \neq \sigma_{2}(i)$ (i.e., the Hamming distance of $\sigma_{1}$ and $\sigma_{2}$ ).

P1 $X_{m_{1}} \sim \mathbf{E}\left(X_{m_{1}}\right) \sim 2^{n}\left(1-2^{-k}\right)^{m_{1}}=e^{\gamma} \mathbf{w h p}$.

$\mathbf{P 2}$ Let $Z_{t}$ denote the number of pairs of satisfying assignments $\sigma_{1}, \sigma_{2}$ for which $h\left(\sigma_{1}, \sigma_{2}\right)=t$. Then whp $Z_{t}=0$ for $0<t<0.49 n$.

Because these properties are not explicitly spelled out in [6], in Section 3 we indicate briefly how they can be demonstrated using the arguments in this reference. We defer their verification until Section 3 and now show how they can be used to prove Theorem 1 .

We generate our instance $I_{m}$ by first generating $I_{m_{1}}$ and then adding the $m-m_{1}$ random clauses $J=\left\{C_{1}, C_{2}, \ldots, C_{m-m_{1}}\right\}$. Suppose that in this case $I_{m_{1}}$ has satisfying assignments $\left\{\sigma_{1}, \sigma_{2}, \ldots, \sigma_{r}\right\}$, where by $\mathbf{P 1}$ we can assume that $r \sim e^{\gamma}$. Now add the random clauses $J$ and let $Y=\mid\left\{i: \sigma_{i}\right.$ satisfies $\left.J\right\} \mid$. We show that for any fixed positive integer $t$,

$$
\mathbf{E}\left(Y_{(t)}\right) \sim e^{-c t}
$$

where $Y_{(t)}=\prod_{j=0}^{t-1}(Y-j)$ signifies the $t$ 'th falling factorial. Thus by standard results, $Y$ is asymptotically Poisson with mean $e^{-c}$ and Theorem 1 follows.

Proof of (3): Since each of the clauses $C_{1}, \ldots, C_{m-m_{1}}$ is chosen independently of all others, we have

$$
\mathbf{E}\left(Y_{(t)}\right)=r_{(t)} \operatorname{Pr}\left(\sigma_{1}, \ldots, \sigma_{t} \text { satisfy } J\right)=r_{(t)} \operatorname{Pr}\left(\sigma_{1}, \ldots, \sigma_{t} \text { satisfy } C_{1}\right)^{m-m_{1}}
$$

Now

$$
\operatorname{Pr}\left(\sigma_{1}, \ldots, \sigma_{t} \text { satisfy } C_{1}\right)=1-\operatorname{Pr}\left(\exists 1 \leq i \leq t: \sigma_{i} \text { does not satisfy } C_{1}\right)
$$

and

$$
\boldsymbol{P r}\left(\exists 1 \leq i \leq t: \sigma_{i} \text { does not satisfy } C_{1}\right) \leq t \operatorname{Pr}\left(\sigma_{1} \text { does not satisfy } C_{1}\right)=\frac{t}{2^{k}}
$$

On the other hand, by inclusion/exclusion

$$
\begin{aligned}
\operatorname{Pr}\left(\exists 1 \leq i \leq t: \sigma_{i} \text { does not satisfy } C_{1}\right) & \\
& \geq \operatorname{Pr}\left(\sigma_{1} \text { does not satisfy } C_{1}\right)-\sum_{1 \leq i<j \leq t} \operatorname{Pr}\left(\sigma_{i}, \sigma_{j} \text { do not satisfy } C_{1}\right) .
\end{aligned}
$$


We then write

$$
\begin{aligned}
& \operatorname{Pr}\left(\sigma_{i}, \sigma_{j} \text { do not satisfy } C_{1}\right) \\
& =\operatorname{Pr}\left(\sigma_{i}, \sigma_{j} \text { do not satisfy } C_{1} \mid \mathbf{P} 2\right) \operatorname{Pr}(\mathbf{P} 2)+\operatorname{Pr}\left(\sigma_{i}, \sigma_{j} \text { do not satisfy } C_{1} \mid \neg \mathbf{P} 2\right) \operatorname{Pr}(\neg \mathbf{P} 2) \\
& =\left(\frac{n-\tau}{2 n}\right)^{k}+o(1) \leq \frac{1}{3^{k}}
\end{aligned}
$$

Finally, going back to (4), we obtain

$$
r_{(t)}\left(1-\frac{t}{2^{k}}\right)^{m-m_{1}} \leq \mathbf{E}\left(Y_{(t)}\right) \leq r_{(t)}\left(1-\frac{t}{2^{k}}+\frac{t^{2}}{3^{k}}\right)^{m-m_{1}} .
$$

Since $t^{2}\left(m-m_{1}\right)=O\left(m-m_{1}\right)=O\left(\omega 2^{k}\right)=o\left(3^{k}\right)$, we get

$$
\mathbf{E}\left(Y_{(t)}\right) \sim r_{(t)}\left(1-\frac{t}{2^{k}}\right)^{m-m_{1}} \sim e^{t \gamma}\left(1-2^{-k}\right)^{t\left(m-m_{1}\right)} \sim e^{-c t},
$$

thereby proving (3).

\section{Verification of $P 1$ and $P 2$}

P1: Let us first compute the expected number $\mathbf{E}\left(X_{m_{1}}\right)$ of satisfying assignments of $I_{m_{1}}$. For any fixed assignment the probability that a single random clause over $k$ distinct variables is satisfied equals $1-2^{-k}$ (because there are $2^{k}$ ways to assign values to the $k$ variables occurring in the clause, out of which $2^{k}-1$ cause the clause to be satisfied). Since the $m_{1}$ clauses are chosen independently, and as there are $2^{n}$ assignments in total, we conclude that $E\left(X_{m_{1}}\right) \sim$ $2^{n}\left(1-2^{-k}\right)^{m_{1}}$. Furthermore, in [6, Section 2] it is shown that $\mathbf{E}\left(X_{m_{1}}^{2}\right) \sim \mathbf{E}\left(X_{m_{1}}\right)^{2}$ and so P1 follows from the Chebyshev inequality.

P2: If $\sigma_{1}, \sigma_{2}$ are two assigments at Hamming distance $h\left(\sigma_{1}, \sigma_{2}\right)=t$, then the probability that either $\sigma_{1}$ or $\sigma_{2}$ does not satisfy a random clause $C_{1}$ is $2^{1-k}-2^{-k}(1-t / n)^{k}$. For the probability that one assignment $\sigma_{i}$ does not satisfy $C_{1}$ is $2^{-k}(i=1,2)$. Moreover, if both $\sigma_{1}$ and $\sigma_{2}$ violate $C_{1}$, then $C_{1}$ is false under $\sigma_{1}$, which occurs with probability $2^{-k}$, and in addition $\sigma_{1}$ and $\sigma_{2}$ assign the same values to all the variables in $C_{1}$, which happens with probability $(1-t / n)^{k}$. Consequently, the expected number of satisfying assignment pairs $\sigma_{1}, \sigma_{2}$ at Hamming distance $t$ in $I_{m_{1}}$ is

$$
F(t)=\mathbf{E}\left(Z_{t}\right)=2^{n}\left(\begin{array}{l}
n \\
t
\end{array}\right)\left(1-2^{1-k}+2^{-k}(1-t / n)^{k}\right)^{m_{1}}
$$

(cf. [6, eq. (5)]). Setting $\rho=m_{1} / n=2^{k}(\ln 2-\gamma / n)+O(1 / n), \tau=t / n$ and taking logarithms, we obtain

$$
\begin{aligned}
f(\tau) & =n^{-1} \ln F(t) \\
& \leq \ln 2-\tau \ln \tau-(1-\tau) \ln (1-\tau)+\rho \ln \left(1-2^{1-k}+2^{-k}(1-\tau)^{k}\right)+O(\tau / n) \\
& \leq \ln 2-\tau \ln \tau-(1-\tau) \ln (1-\tau)-2^{-k} \rho\left(2-(1-\tau)^{k}\right)+O(\tau / n) \\
& =\ln 2-\tau \ln \tau-(1-\tau) \ln (1-\tau)-(\ln 2-\gamma / n)\left(2-(1-\tau)^{k}\right)+O\left(\left(\tau+2^{-k}\right) / n\right) .
\end{aligned}
$$


To show that $\sum_{1 \leq t \leq 0.49 n} F(t)=o(1)$, we consider three cases:

Case 1: $n^{-1} \leq \tau \leq \ln ^{-1.1} n$. Since $(1-\tau)^{k}=1-k \tau+O\left(k^{2} \tau^{2}\right),-(1-\tau) \ln (1-\tau) \leq \tau$, and $k \ln 2=\ln n+\omega \ln 2$, we obtain via (5),

$$
\begin{aligned}
f(\tau) & \leq \tau(1-\ln \tau)-k \tau \ln 2(1-O(k \tau))+2 \gamma / n \\
& \leq \tau(1+\ln n-(\ln n+\omega \ln 2)+o(1)) \\
& \leq-\tau \omega / 2 .
\end{aligned}
$$

Consequently,

$$
\sum_{1 \leq t \leq n \ln ^{-1.1} n} F(t)=\sum_{1 \leq t \leq n \ln ^{-1.1} n} \exp (n f(t / n)) \leq \sum_{1 \leq t \leq n \ln ^{-1.1} n} \exp (-\omega t / 2)=o(1) .
$$

Case 2: $\ln ^{-1.1} n<\tau \leq k^{-1} \ln \ln n$. We have, for large $n$,

$$
-\tau \ln \tau-(1-\tau) \ln (1-\tau) \leq \tau(1-\ln \tau) \leq \frac{(1+\ln k) \ln \ln n}{k} \leq k^{-\frac{1}{2}} \leq \ln ^{-\frac{1}{2}} n .
$$

On the other hand, for large $n$,

$$
(1-\tau)^{k} \leq \exp (-k \tau) \leq \exp \left(-k \ln ^{-1.1} n\right) \leq 1-\ln ^{-0.1} n
$$

Thus, from (5),

$$
f(\tau) \leq \ln 2+\ln ^{-\frac{1}{2}} n-\ln 2-\frac{\ln 2}{\ln ^{0.1} n} \leq-\frac{1}{2} \ln ^{-0.1} n .
$$

Hence, if $n \ln ^{-1.1} n<t \leq n k^{-1} \ln \ln n$, then $F(t) \leq \exp \left(-\frac{1}{2} n \ln ^{-0.1} n\right)$, which implies

$$
\sum_{n \ln ^{-1.1}} F(t)=o(1) .
$$

Case 3: $k^{-1} \ln \ln n<\tau \leq 0$.49. Since $\tau \gg k^{-1}$, we have $(1-\tau)^{k}=o(1)$, whence

$$
(\ln 2-\gamma / n)\left(2-(1-\tau)^{k}\right) \sim 2 \ln 2 .
$$

Furthermore, as the entropy function $\tau \mapsto-\tau \ln \tau-(1-\tau) \ln (1-\tau)$ is increasing on $\left[0, \frac{1}{2}\right]$, we have

$$
\ln 2-\tau \ln \tau-(1-\tau) \ln (1-\tau) \leq \ln 2-0.49 \ln (0.49)-0.51 \ln (0.51)<1.9998 \ln 2 .
$$

Hence, $f(\tau) \leq-0.0001$. Therefore, $F(t) \leq \exp (-0.0001 n)$, and thus

$$
\sum_{n k^{-1} \ln \ln n<\tau \leq 0.49 n} F(t)=o(1) .
$$

Combining (6)-(8), we conclude that $\sum_{1 \leq t \leq 0.49 n} F(t)=o(1)$. Thus, whp $Z_{t}=0$ for all $1 \leq t \leq 0.49$. 


\section{Conclusion}

It is instructive to compare the $k$-SAT problem with $k>\log _{2} n+\omega$, which we have studied in the present paper, with the case of constant $k$. We have shown that for $k>\log _{2} n+\omega$ in the regime $m / n-2^{k} n \ln 2=\Theta\left(2^{k}\right)$ the number of satisfying assignments is asymptotically Poisson. The basic reason is that the mutual Hamming distance of any two satisfying assignments is about $n / 2$ (cf. property P2). Hence, the set of all satisfying assignments consists of isolated points in the Hamming cube, which are mutually far apart. By contrast, in the case of constant $k$ in the near-threshold regime the set of satisfying assignments seems to consist of larger "cluster regions" (cf. Achlioptas and Ricci-Tersenghi [2] and Krzakala, Montanari, Ricci-Tersenghi, G. Semerjian, and L. Zdeborova [9]).

In Theorem 1 we assume that $\omega=k-\log _{2} n=o(\ln n)$. While this assumption eases some of the computations, the result (and the proof technique) can be extended to larger values of $k$. Nevertheless, the case $k<\log _{2} n$ appears to us to be a more interesting problem.

\section{References}

[1] D. Achlioptas and C. Moore: Random $k$-SAT: two moments suffice to cross a sharp threshold. SIAM Journal on Computing 36 (2006) 740-762.

[2] D. Achlioptas and F. Ricci-Tersenghi: On the solution-space geometry of random constraint satisfaction problems. Proceedings of the 38th Annual ACM Symposium on Theory of Computing (2006) 130-139.

[3] D. Achlioptas and Y. Peres, The threshold for random $k$-SAT is $2^{k}-\log 2-O(k)$, Journal of the American Mathematical Society, 17 (2004), 947-973.

[4] O. Dubois, Y. Boufkhad and J. Mandler, Typical random 3-SAT formulae and the satisfiability threshold, Proceedings of the Eleventh Annual ACM-SIAM Symposium on Discrete Algorithms (2000) 126-127.

[5] E. Friedgut, Sharp thresholds of graph properties, and the $k$-sat problem. With an appendix by Jean Bourgain. Journal of the American Mathematical Society 12 (1999) 1017-1054.

[6] A.M. Frieze and N. Wormald, Random $k$-SAT: A tight threshold for moderately growing $k$, Combinatorica 25 (2005) 297-305.

[7] M.T. Hajiaghayi and G.B. Sorkin, The satisfiability threshold of random 3-SAT is at least 3.52. IBM Research Report RC22942 (2003)

[8] A.C. Kaporis, L.M. Kirousis, and E.G. Lalas: Selecting complementary pairs of literals. Electronic Notes in Discrete Mathematics 16 (2003)

[9] F. Krzakala, A. Montanari, F. Ricci-Tersenghi, G. Semerjian, L. Zdeborova, Gibbs states and the set of solutions of random constraint satisfaction problems. Preprint (arXiv:condmat/0612365). 